\title{
AntiGroupWare and Second Messenger
}

\section{I Norton, J M DiMicco, R Caneel and D Ariely}

Decision-making in groups has great potential due to the possibilities for pooling ideas and sharing knowledge, but also great drawbacks due to the social pressures inherent in these situations that can limit free exchange of these ideas and knowledge. This paper presents two technology-based approaches to improving group decision-making, Second Messenger and AntiGroupWare. Second Messenger — a system that encourages groups to change their interaction styles during meetings - is designed to improve meetings, while AntiGroupWare - an on-line polling system that allows companies to gather information through flexible, iterative polling of its employees - is designed to avoid them altogether.

\section{Introduction}

It was the morning of March 19, 2003 in the White House Situation Room, just hours before President Bush's 48-hour ultimatum to Saddam Hussein to get out of Iraq was to expire. President Bush had just polled his war council for any last-minute reservations about the war plan. Hearing none, he issued the 'execute' command to General Tommy R Franks.

The New York Times, April 14th 2003

Organisations are called upon to make decisions almost continuously, and one of the most common models for doing so utilises a format with which most are all too familiar gathering relevant individuals together in a meeting to discuss the issues at hand and reach a decision to be implemented. This approach seems at first glance to be an ideal strategy, but 50 years of research on the drawbacks of making decisions in groups suggests that this model - despite its widespread use - can lead to suboptimal decision-making [1]. In the example above, two factors stand out for their potentially adverse impact on decision-making in groups - authority structures and conformity pressures. As the clear authority, Bush spoke first, making his position clear, and then polled his subordinates for their opinions. Additionally, by asking each person for their opinion one-by-one, the pressure for the last Cabinet member to agree with the plan increased. Thus while it appears that the decision to attack Iraq was a unanimous decision made by a group of highly skilled individuals, the pressures on this group to agree with the authority figure and conform to the majority opinion may have undermined its ability to make optimal decisions.
This is just one example of where group deliberation can result in poor decision-making. In one of the most infamous episodes in American foreign policy, John F Kennedy and his advisors, after extensive group discussion of the plan, signed off on the 'Bay of Pigs' invasion of Cuba in 1961, a plan that had virtually no chance of success [2].

\section{sadly, the intuitions about the benefits of group decision-making seem to be overly optimistic}

In one of the most infamous cases of American corporate chicanery, nearly every board vote taken at the Enron Corporation was unanimous [3] - despite the fact that Enron's policies were disastrous. How can groups of qualified, intelligent individuals make such suboptimal decisions when gathered together? How is it that pooling the knowledge and expertise of individuals sometimes fails to improve decisionmaking? Sadly, the intuitions about the benefits of group decision-making seem to be overly optimistic; groups sometimes bring the worst out of individuals.

This paper presents two systems, Second Messenger and AntiGroupWare, designed to remedy these problems of decision-making in groups. Second Messenger focuses on improving decision-making during meetings, while AntiGroupWare focuses on improving decision-making out of meetings, allowing meetings to be avoided altogether. In the 
following sections, we review the literature on group biases, discuss the potential for technology to improve these biases, and then describe how Second Messenger and AntiGroupWare might help.

\section{Two key problems with groups - authority and conformity}

Two factors particularly likely to have an adverse impact on decision-making in meetings are authority structures and conformity pressures. Those in positions of authority or influence may or may not have greater expertise or knowledge than others, but due to the tendency of individuals to defer to authority figures (following their advice and endorsing their recommendations even when these decisions are flawed or even harmful), those in positions of power can exert inordinate influence over the tone of the discussion and the eventual outcome. A classical illustration of the power of authority was provided in a set of famous experiments by Stanley Milgram, where individuals were willing to administer what they thought were harmful, or even fatal, electric shocks to other participants simply because a 'scientist' in a white lab coat told them to do so [4]. In the real-world setting of airplane flight crews, the term 'captainitis' was coined to describe the many near-disaster airplane incidents in which a member of the flight crew noticed a problem but did not to mention it to the captain, presumably because they did not trust their own judgement over the captain's [5]. Though non-authority figures often have information and expertise that is relevant to the decision at hand, concerns regarding authority can prevent individuals from airing their views publicly. To the extent that it inhibits this disclosure of information, the presence of authority figures can have a seriously adverse impact upon group decision-making.

\section{the presence of authority structures and conformity pressures can lead to less information-sharing and less discussion of conflicting viewpoints}

Even in the absence of authority figures, the mere presence of others can have unexpected and negative effects on group decision-making. As adolescents know all too well, the opinions, preferences, and behaviour of one's peers can have an inordinate impact on personal choices. When several individuals express one opinion in a meeting, other individuals can conform to this emerging group norm, even when that opinion is objectively wrong. Solomon Asch demonstrated this tendency in a set of classic experiments in which participants heard a series of other people give what was clearly a wrong answer to a simple judgement task. Asch's results revealed that the more people who had given the wrong answer, the more likely the study participant - the only person who was not in league with the experimenter — was to also give the wrong answer [6]. In some cases, up to $75 \%$ of people would give the wrong answer, simply to fit in with their peers. Because real life decisions are, if anything, even more ambiguous than the ones Asch used in his experiments, conformity pressures can be powerful in inducing individuals to acquiesce publicly to decisions with which they disagree privately.

In summary, the presence of authority structures and conformity pressures can lead to less information-sharing and less discussion of conflicting viewpoints, resulting in suboptimal decisions. This is not to say that all social influence in groups will have negative consequences, of course - it is sometimes the case that those in authority are present because they may be better qualified to guide discussion and shape policy, or that consensus is dictated not by conformity but by the facts. Rather, what the present paper seeks to address are those cases where the quality of the group decision is adversely impacted by social pressures of which the group is unaware and in response to which it may be acting blindly, and thus succumbing to the negative effects of group interaction.

\section{Technology and group decision-making}

The underlying assumption behind both Second Messenger and AntiGroupWare is that technology can be used to improve group decision-making, a concept that has been increasingly studied over the last twenty years. When technology is introduced as a communications medium between group members, the way we communicate fundamentally changes, and in some cases this can create additional group process problems. For example, videoconferencing is commonly used for meetings within companies, yet studies have shown that there is less trust between individuals and that it is more difficult to establish common ground over video than in faceto-face interactions [7-9]. When communication is textbased, such as with chat, e-mail or, increasingly, instantmessenger, decision-making tasks take more time and produce lower rates of task accuracy [10-12].

Text-based applications also cause individuals to make more declarative position statements and less information-based statements [13], making productive information-based meetings more difficult to hold. In the specific areas of group polarisation and information sharing, which relate back to our issues of authority and conformity, it has been shown that groups can experience more group polarisation [10,12] and less information sharing [14] when communicating in a textbased environment.

At the same time, some research shows that technology when used appropriately - can help improve group performance, by minimising inhibitory social pressures [15]. In systems that allow groups to anonymously interact and contribute to a brainstorming task, studies have shown marked improvement in systems that are anonymous over those that identify the users $[16,17]$. Along these lines, we outline below two systems, Second Messenger and AntiGroupWare, that attempt to utilise the positive aspects of technology for the purpose of improving group decisionmaking. 


\section{In-meeting decisions - Second Messenger}

Second Messenger is a system that focuses on encouraging a group to change its interaction during a meeting, based on the idea that making a group aware of its potentially flawed interaction while it is taking place affords the group the opportunity to correct its behaviour. When interacting face-toface, individuals utilise their natural strengths in communicating opinions and intentions, yet also naturally overly rely on the group's dominating opinions, as already discussed. We postulate that a system observing the natural dynamics of a group interaction can detect skewed group processes and can then influence the group, through visual displays of social information, to alter its interaction to focus more successfully on the breadth of ideas in a discussion.

As an initial proof-of-concept and to explore the possibilities for the type of system proposed, the Second Messenger application [18] augments a face-to-face interaction by providing a real-time visual summary of the verbal comments made during a group meeting. Second Messenger is built around a client-server architecture where each group member wears a microphone that sends his/her spoken dialogue to a client running IBM's ViaVoice, using a trained voice model to recognise individual speech. When the user speaks, the transcribed words are sent to the server for analysis and filtering. After filtering for accuracy and uniqueness, the text phrases appear on the shared display and can be manipulated and organised using a mouse, making it a tool for organising the group discussion (see Fig 1 for a screenshot). In addition to being a tool though, Second Messenger attempts to increase the amount of discussion around diverse viewpoints by increasing the visibility of the comments made by group members who speak less frequently and by filtering out the comments of group members who verbally dominate.

The next step of this research was to run a controlled behavioural experiment to understand how a simple display of speaker participation could have an impact on the behaviour of a group in a decision-making task [19]. The purpose of this was to begin exploring the area of how to influence, and improve, the behaviour of groups through the use of social information displays. The interface for the experiment

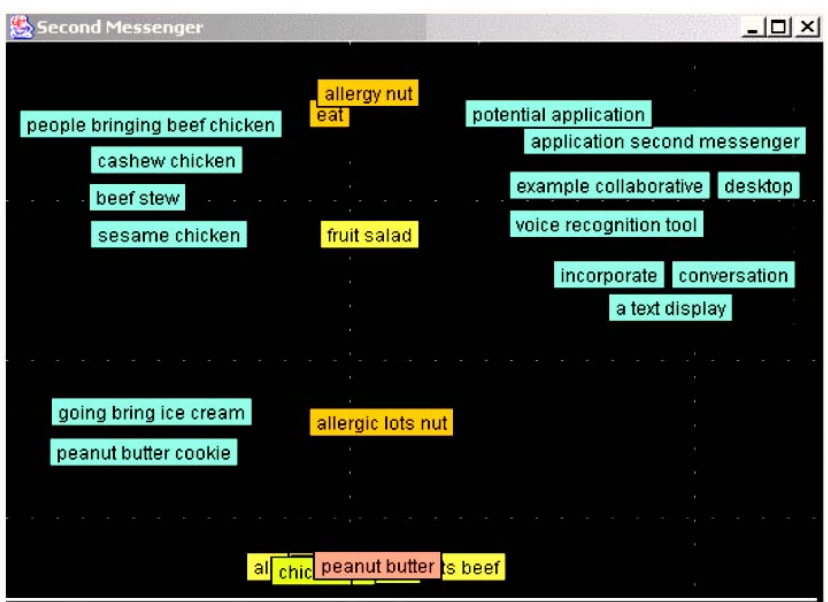

Fig 1 Second Messenger's shared display. removed the textual content of Second Messenger and instead displayed the quantity of spoken comments made by each participant. As a group interacts, the display dynamically adjusts the bars of a histogram to indicate the relative participation rates (see Fig 2 for a screenshot). The system is built again with a client/server architecture, where each client machine determines when someone is speaking by detecting the sound level from individual microphones.

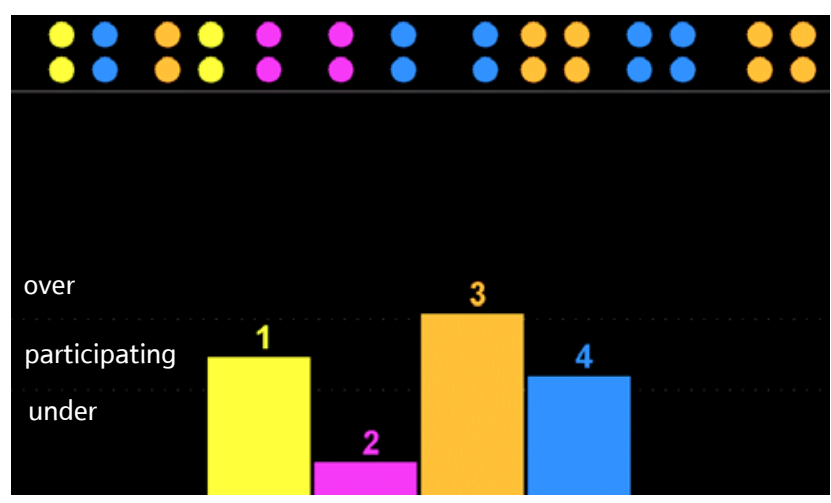

Fig 2 Histogram display of speaker participation.

The behavioural experiment's protocol was based on an information-sharing task previously used by Hollingshead [14] in which each subject group was given a task to solve, the successful completion of which depended on the amount of information shared by the group. The study's control condition had groups complete two tasks with no technological assistance; the experimental condition had the display projected on to the wall during the second of two tasks.

The main finding from the study was that the overparticipators ${ }^{1}$ decreased the amount they spoke in the experimental condition significantly more so than those in the control condition ( $p<.05^{*}$, t-test of independent samples) and the under-participators did not change the amount they spoke in the experimental condition $\left(p<.05^{*}\right.$, paired t-test), in contrast to the under-participators in the control condition who did significantly increase the amount they spoke $\left(p<.05^{*}\right.$, paired t-test). Figure 3 shows these results graphically.

These results confirmed our hypothesis that a display of social information has an impact upon the behaviour of individuals in a group decision-making setting, specifically encouraging over-participators to temper their comments. The finding that under-participators did not increase the amount they spoke was unexpected and has lead us in new directions for building private information displays.

The two outcomes of the Second Messenger project are a demonstration that a real-time tool for group collaboration using speech-recognition can be incorporated into a meeting, and that a simple display of information about group dynamics

\footnotetext{
1 The definitions for over- and under-participators were defined by measuring the participation of subjects during a preliminary task and categorizing subjects who participated above the (mean + one standard deviation) as 'over' and those who participated below the (mean - one standard deviation) as 'under'.
} 


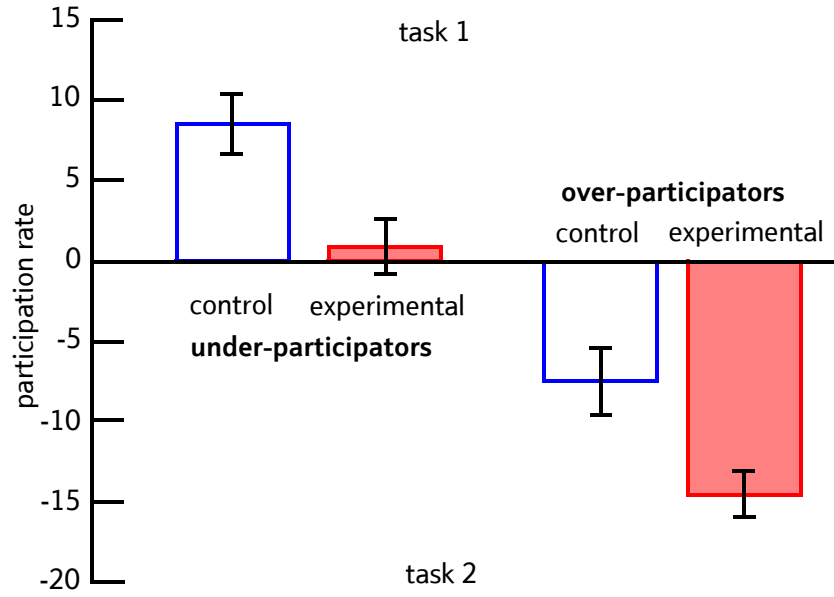

Fig 3 Changes in participation rates by participation category and condition.

does have an impact on the behaviour of a group. Our planned future work is to continue to build visual displays of ongoing social interaction and develop a deeper understanding of how these displays encourage and discourage certain behaviours. Our goal is to produce a set of interfaces that increase the diversity of a group's discussion and assist in improving the quality of the interaction.

\section{Out-of-meeting decisions - AntiGroupWare}

AntiGroupWare is an alternative approach to improving decision-making that enables groups to avoid meeting altogether. The AntiGroupWare application is an on-line polling system that companies can use to make decisions through flexible, iterative polling of its employees. Thus the system is designed to tap into organisational knowledge, which has received increasing attention as a useful source of information for corporations. Aggregation of estimates made by Hewlett-Packard employees were shown to be a better predictor of the value of HP stock than the predictions of, for example, the highly trained analysts hired by $\mathrm{HP}$, and the 'average Americans' who participate in the lowa Electronic Markets have proved to be more accurate than experts in forecasting the outcomes of US presidential campaigns. To harness the strength of collective knowledge and to improve upon existing models, AntiGroupWare has three key features - flexible voting, iterative voting, and proxy voting.

\subsection{Flexible voting}

When polling is limited to basic voting procedures, such as allowing single 'Yes/No' responses to single issues, the effectiveness of the poll is limited. AntiGroupWare provides a much-increased level of flexibility in collecting responses. Specifically, the AntiGroupWare system allows individuals to rate multiple options on multiple dimensions. For example, a proposal can be rated for its maximum benefit and its risk. As a result, the separate evaluation of these two estimates offer a fuller sense of the expected payoff of the proposal than a simple 'Yes/No' would have. AntiGroupWare also allows individuals to rank multiple options, so organisations can assess support for a fuller set of alternatives (for example it is possible that all individuals differ on their most preferred option but they all agree on the second best option or the least preferred option). An example of such a method is known as the Borda preferendum, in which ranks are associated with graduated points (e.g. 1st ranked options gets 15 points, 2 nd ranked gets 10,3 rd gets 8 , etc), a system that has been used effectively in other domains (e.g. professional sports 'Most Valuable Player' awards).

\subsection{Iterative voting}

Another feature of the system is that polls can allow for iterative voting. An advantage of this is that individuals can dynamically react to the information available in others' responses. This approach is in contrast to the way voting is usually carried out, which can be characterised as a 'one-shot simultaneous process' much like sealed-bid auctions. This function bears some resemblance to the Delphi technique developed at the Rand Corporation [20], a multiple-round process by which managers can pool the opinions of selected individuals. These procedures were often time-consuming, involving multiple rounds of paper questionnaires, an issue avoided by using on-line mechanisms. The nominal group technique [21], in which participants meet in groups but first write their opinions about issues privately before sharing with other group members, is another system designed to improve group-decision-making; again, however, while the technique has benefits, it requires lengthy meetings, and a moderator. The ability to express opinions iteratively - with little cost and time - makes AntiGroupWare resemble an informationsorting mechanism such as the stock market or an open auction. To illustrate this point, consider an open auction, where the purpose is price discovery and there are benefits in letting some people voice their opinions (prices) early, letting others observe these opinions and then considering this new information together with their prior opinions. In particular, this process can help in information discovery in cases where people can get additional insights from the opinions of others. When bidding on an antique divan, for example, the fact that others value it highly decreases the probability that the divan is a fake. Thus the means by which the opinions of others are revealed can be beneficial to decision makers. We propose that similar systems can be useful for voting and opinion mechanisms more generally, in those cases where the information contained in the opinions of others can inform one's own understanding. AntiGroupWare has the facility to allow dynamic voting that lasts over multiple stages where individuals can revisit their votes, see where the winds are blowing in the overall decision, and if they so desire change their opinions. This process, while more involved than oneshot voting, clearly has the potential to improve decision quality.

\subsection{Proxy voting}

A final feature of AntiGroupWare is its ability for individuals to assign their votes to other individuals who may be more knowledgeable, an opportunity usually restricted to management. First, the system allows managers to assign greater weight to the votes of those individuals deemed to be most qualified. More interestingly, AntiGroupWare also allows 
individuals themselves to assign their votes (proxy-voting) to someone of their choosing, if they feel that another person would be better equipped to make a decision in some domain. For example, experts in recycling, who are recognised by their peers as such, might get assigned voting rights by their peers for topics related to recycling. Importantly, in order to maintain anonymity, those who have been assigned additional weight make their choices or judgements without the knowledge of their additional power of influence - or the source(s) of that increased influence. This system thus has the potential to tap into organisational knowledge not captured on organisational flow charts (and thus not necessarily available to managers), and better utilises organizational knowledge by allocating decision-making authority to the best-qualified individuals.

It will not always be the case that individuals know who is most knowledgeable, or that they will assign their votes in the most optimal manner, yet results such as those at Hewlett Packard and in the lowa Electronic Markets suggest that this kind of knowledge can be extremely useful.

\subsection{Summary}

The anonymity inherent in AntiGroupWare addresses the negative impact of both authority and conformity - the former by ensuring that management cannot monitor which employee has voted for which proposal, and the latter by allowing for votes to be taken in private where people are licensed to express their true opinions. The iterative voting functionality allows for some social influence to occur (as people may change their votes to match a growing consensus) but because the voting is anonymous the social influence caused by iterative voting may be more informational in nature - as people gather information about others' opinions, they may come to change their opinion not due to social pressure but due to new information revealed through the voting process.

Finally, the proxy voting functionality addresses issues of authority by standing authority on its head, and allowing employees to designate authority as they choose, rather than being bound by institutional authority structures.

\section{'... give ourselves time to develop disagreement and perhaps gain some understanding of what the decision is all about ...' Alfred Sloan}

The AntiGroupWare application is currently operational with two of the three features outlined above - flexible and iterative voting - and we are planning to test the system at Ford Motor Company's new product development sector, to assist Ford in gaining better feedback from employees about new models and options for product lines.

\section{Conclusions}

Alfred Sloan, who ran General Motors from 1923 to 1956, once said during a meeting: 'Gentlemen, I take it that we are all in complete agreement on the decision here. Then, I propose that we postpone further discussion... to give ourselves time to develop disagreement and perhaps gain some understanding of what the decision is all about' [3]. Alfred Sloan was not a management genius for nothing. Years later we are following in his footsteps with an increased understanding of the reasons underlying poor group decisionmaking, with more advanced approaches for solving them. The technology-based approaches to group decision-making presented here, Second Messenger and AntiGroupWare, can minimise group decision biases while at the same time improving data aggregation more generally both in and out of meetings. Second Messenger allows for better sharing of information, and thus more optimal decisions, while AntiGroupWare is designed to improve and streamline decision-making, at the same time sparing people lengthy, and often unproductive meetings.

\section{Acknowledgements}

The work described in this paper has been supported by the Digital Life and information:organized consortia at the Media Lab. Additional thanks go to the extremely helpful anonymous reviewers.

\section{References}

1 Davis J H: 'Some compelling intuitions about group consensus decisions, theoretical and empirical research, and interpersonal aggregation phenomena: Selected examples, 1950-1990', Organizational Behavior and Human Decision Processes, 52 , pp 3-38 (1992).

2 Janis I: 'Groupthink', Boston, MA, Houghton Mifflin (1982).

3 Surowiecki J: 'Board Stiffs', The New Yorker (8 March 2004).

4 Milgram S: 'Obedience to authority: An experimental view', New York, Harper and Row (1974).

5 Foushee M C: 'Dyads and triads at 35,000 feet: Factors affecting group process and aircraft performance', American Psychologist, 39, pp 885-893 (1984).

6 Asch S E: 'Studies of independence and conformity: I. A minority of one against a unanimous majority', Psychological Monographs, 70, No 416, p 70 (1956).

7 Isaacs E and Tang J C: 'What video can and cannot do for collaboration', Multimedia Systems, 2, pp 63-73 (1994).

8 O'Malley C, Langton S, Anderson A, Doherty-Sneddon G and Bruce V: 'Comparison of face-to-face and video mediated interaction', Interacting with Computers, 8 , pp 177-192 (1996).

9 Rocco E: 'Trust breaks down in electronic contexts but can be repaired by some initial face-to-face contact', Conference on Human Factors in Computing Systems (CHI98), Los Angeles (1998).

10 Kiesler S and Sproull L: 'Group decision making and communication technology', Organizational Behavior and Human Decision Processes, 52, pp 96-123 (1992).

11 Ochsman R B and Chapanis A: 'The effects of 10 communication modes on the behavior of teams during co-operative problemsolving', International Journal of Man-Machine Studies, $\underline{6}$, pp 579-619 (1974). 
12 Siegel J, Dubrovsky V, Kiesler S and McGuire T W: 'Group processes in computer-mediated communication', Organizational Behavior and Human Decision Processes, 37, pp 157-187 (1986).

13 Reid F, Ball L et al: 'Styles of group discussion in computermediated decision making', British Journal of Social Psychology, 36, pp 241-262 (1997).

14 Hollingshead A B: 'Information suppression and status persistence in group decision-making - the effects of communication media', Human Computer Research, 23, pp 193-219 (1996).

15 Dennis A R, George J F, Jessup L M, Nunamaker J F and Vogel D R: 'Information technology to support electronic meetings', MIS Quarterly, 12, pp 591-624 (1998).

16 Connolly T, Jessup L M and Valacich J S: 'Effects of anonymity and evaluative tone on idea generation in computer-mediated groups', Management Science, 36, pp 97-120 (1990).

17 Jessup L M, Connolly T and Galegher J: 'The effects of anonymity on GDSS group process with an idea-generating task', MIS Quarterly, 14, pp 313-321 (1990).

18 DiMicco J M and Bender W: 'Second Messenger: Increasing the Visibility of Minority Viewpoints with a Face-to-face Collaboration Tool', Proceedings of the ACM Conference on Intelligent User Interfaces (2004).

19 DiMicco J M, Pandolfo A and Bender W: 'Influencing Group Participation with a Shared Display', Proceedings of the ACM Conference on Computer Supported Cooperative Work (CSCW 2004) (in submission).

20 Brown B B: 'Delphi Process: A methodology used for the elicitation of opinions of experts', Santa Monica, CA, Rand Corporation (1968).

21 Delbecq A L, Van de Ven A H and Gustafson D H: 'Group techniques for program planning', Glenview, IL, Scott, Foresman (1975).

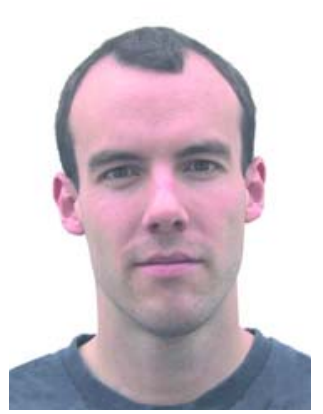

Michael I Norton is a post-doctoral fellow at MIT's Sloan School of Management and the MIT Media Lab. He received his PhD in social psychology from Princeton University.

His research interests include judgement and decision-making; the nature and consequences of political correctness, life satisfaction and well-being (in domains ranging from religious practices to consumer behaviour), and political debate strategy.

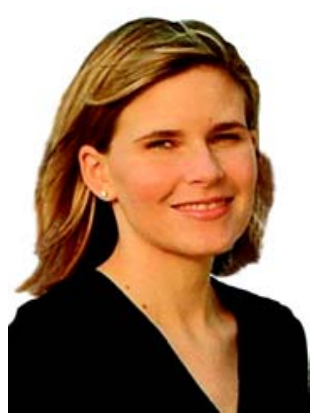

Joan Morris DiMicco is a PhD student in the Electronic Publishing group at the Media Lab. She holds a BS in applied mathematics from Brown University and an MS in media arts and sciences from MIT. Her master's research focused on dynamic pricing in agent-driven marketplaces. Prior to the Media Lab, she worked in the 'dot.com' domain, introducing the Internet's first secure payment system, created at First Virtual Inc, and next at Open Sesame, where she built Web sites that learned users' preferences by observing their on-line behaviour. Her doctoral research continues to explore how technology can observe and recognise patterns in human behaviour, and in particular how this information can be used to assist groups in improving their interaction and decision-making processes in face-to-face settings.

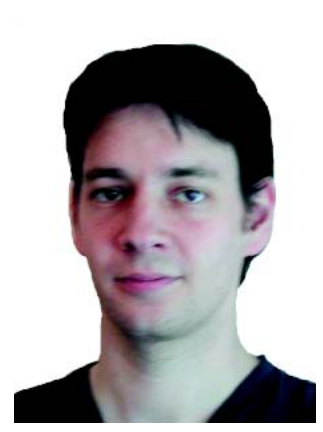

Ron Caneel is a master's student in MIT's program in media arts and sciences.

He received an MS in computer science from the Swiss Federal Institute of Technology (ETH) in 2001 with a minor in psychology from the University of Zurich.

His research interests are group decisionmaking and modelling and analysing group interactions.

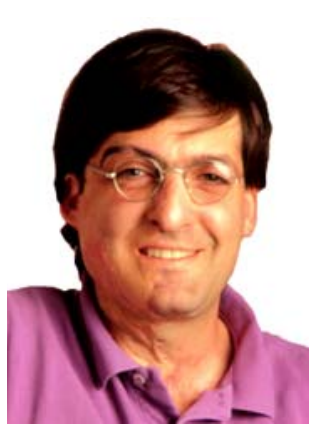

Dan Ariely holds a joint appointment between MIT's Program in Media Arts and Sciences and Sloan School of Management, and is the principal investigator of the Lab's eRationality group. He is interested in issues of rationality, irrationality, decision-making, behavioural economics, and consumer welfare. Projects include examinations of on-line auction behaviours, personal health monitoring, the effects of different pricing mechanisms, and the development of systems to overcome day-to-day irrationality. He received a $\mathrm{PhD}$ in marketing from Duke University, an $\mathrm{MA}$ and $\mathrm{PhD}$ in cognitive psychology from the University of North Carolina at Chapel Hill, and a BA in psychology from Tel Aviv University. 